\title{
A/G polymorphism of matrix metalloproteinase 7 gene promoter region and cancer risk: A meta-analysis
}

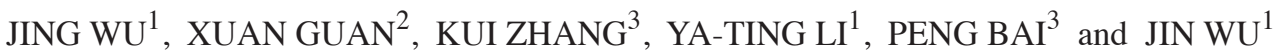 \\ ${ }^{1}$ Department of Forensic Biology, West China School of Preclinical and Forensic Medicine, Sichuan University, \\ Chengdu, Sichuan 610041; ${ }^{2}$ School of Basic Medical Sciences, Chengdu Medical College, Chengdu, Sichuan 610500; \\ ${ }^{3}$ Laboratory of Molecular Translational Medicine, West China Second University Hospital, \\ Sichuan University, Chengdu, Sichuan 610041, P.R. China
}

Received March 03, 2013; Accepted July 08, 2013

DOI: $10.3892 /$ br.2013.131

\begin{abstract}
This meta-analysis was conducted to evaluate the effect of the matrix metalloproteinase 7 (MMP7)-181A/G polymorphism on cancer risk. Twenty-seven case-control studies were identified via a literature search through PubMed, Web of Science and China National Knowledge Infrastructure databases. Odds ratios (ORs) and corresponding 95\% confidence intervals (CIs) were applied to assess the strength of the association between MMP7-181A/G polymorphism and cancer risk. The 27 studies were further assessed according to Hardy-Weinberg equilibrium (HWE) and Hardy-Weinberg disequilibrium (HWD), with 24 case-control studies found to be under HWE. A significant association was observed between MMP7-181A/G polymorphism and increased cancer risk (cervical and other types of cancer) in Asian, but not in European populations.
\end{abstract}

\section{Introduction}

The processes of tumorigenesis and metastasis involve cancer cell migration and penetration through the extracellular matrix (ECM) (1). Matrix metalloproteinases (MMPs) are a family of highly conserved zinc-dependent proteolytic enzymes that are able to degrade essentially all ECM components and regulate diverse cell behaviors (2). MMPs play pivotal roles in physiological ECM remodeling, such as tissue regeneration in pregnancy, wound healing and angiogenesis (3). MMPs are also involved in pathological conditions, such as cancer, arthritis, autoimmune diseases and atherosclerosis (4).

Matrix metalloproteinase 7 (MMP7) (punctuated metalloproteinase-1, PUMP-1, matrilysin) is coded by a gene localized

Correspondence to: Professor Jin Wu, Department of Forensic Biology, West China School of Preclinical and Forensic Medicine, Sichuan University, 17 South Renmin Road, Chengdu, Sichuan 610041, P.R. China

E-mail:wujin2028@163.com

Key words: polymorphism, matrix metalloproteinase, cancer risk, meta-analysis on chromosome 11q21-q22. MMP7 is capable of degrading elastin, fibronectin, proteogylcans and type IV collagen (5) and of cleaving non-matrix substrates of the cell surface, such as E-cadherin, pro-tumor necrosis factor and Fas ligand. MMP7 is predominantly expressed in the epithelium of various organs under physiological conditions and may be overexpressed in a variety of cancers, such as cancers of the colorectum, esophagus, stomach, kidney and breast $(6,7)$.

Numerous molecular epidemiological studies on the association of MMP7 polymorphisms with cancer susceptibility have been conducted. However, the association between MMP7-81A/G polymorphism and cancer risk has not been elucidated. Therefore, a system review and meta-analysis was performed.

\section{Materials and methods}

Search strategy and eligibility criteria. Two investigators (J. Wu and X. Guan) independently conducted key word searches in PubMed, Web of Science and the China National Knowledge Infrastructure databases to identify all eligible studies between 2000 and 2013. The following terms were used: 'MMP7' or 'matrix matalloproteinase 7' and 'polymorphism' and 'cancer', 'tumor', 'neoplasm' or 'carcinoma' (last search update, February 20th, 2013). References cited in the publications were also screened by hand. The following criteria were required to be met: i) the publication was a case-control study; ii) the study evaluated the association between MMP7-181A/G polymorphism and cancer; iii) odds ratios (ORs) or available data for their calculation were reported; and iv) the study was published in English or Chinese.

Data extraction. The following basic data were collected: first author, publication year, cancer type and ethnicity of study populations. Two investigators conducted data extraction independently and discrepancies were resolved through discussions.

Statistical analysis. Odds ratios (ORs) and corresponding 95\% confidence intervals (CIs) were calculated to assess the association between MMP7-181A/G polymorphism and cancer risk (8). Stratified analyses were also performed by cancer type (if one cancer type was contained in less than three single 
Table I. Characteristics of studies included in the meta-analysis.

\begin{tabular}{|c|c|c|c|c|c|c|c|}
\hline Author & Year & Country & Ethnicity & Cancer type & $\begin{array}{l}\text { Sample size } \\
\text { (case/control) }\end{array}$ & $\mathrm{P}_{\mathrm{HWE}}$ & Refs. \\
\hline Ghilardi et al & 2003 & Italy & European & Colorectal & $58 / 111$ & 0.129 & $(27)$ \\
\hline Li et al & 2008 & China & Asian & Gastric & $338 / 380$ & 0.999 & $(30)$ \\
\hline Ohtani et al & 2009 & Japan & Asian & Colorectal & $119 / 67$ & 0.421 & $(24)$ \\
\hline Wu et al & 2011 & China & Asian & Cervical & $217 / 190$ & 0.010 & $(36)$ \\
\hline Kubben et al & 2006 & The Netherlands & European & Gastric & $79 / 169$ & 0.000 & (34) \\
\hline Vairaktaris et al & 2007 & Germany/Greece & European & Oral & $159 / 120$ & 0.018 & $(35)$ \\
\hline Li et al & 2006 & China & Asian & Ovarian & $138 / 160$ & 0.714 & $(29)$ \\
\hline Woo et al & 2007 & Korea & Asian & Colorectal & $185 / 304$ & 0.232 & $(25)$ \\
\hline Fang et al & 2010 & China & Asian & Colorectal & $237 / 252$ & 0.315 & $(18)$ \\
\hline Lu et al & 2006 & China & Asian & Adult astrocytoma & $221 / 366$ & 0.319 & $(6)$ \\
\hline de Lima et al & 2009 & Brazil & other & Colorectal & $108 / 113$ & 0.488 & $(28)$ \\
\hline Dziki et al & 2011 & Poland & European & Colorectal & $184 / 205$ & 0.295 & $(17)$ \\
\hline Lievre et al & 2006 & France & European & Colorectal & $596 / 565$ & 0.098 & $(20)$ \\
\hline Singh et al & 2008 & India & Asian & Cervical & $150 / 162$ & 0.750 & $(22)$ \\
\hline Srivastava et al & 2010 & India & Asian & Bladder & $200 / 200$ & 0.810 & (19) \\
\hline Sugimoto et al & 2008 & Japan & Asian & Gastric & $160 / 156$ & 0.332 & $(16)$ \\
\hline Yi et al & 2010 & Taiwan & Asian & Endometrial & $118 / 229$ & 0.931 & $(21)$ \\
\hline Qiu et al & 2008 & China & Asian & Hepatocellular & $425 / 475$ & 0.338 & $(23)$ \\
\hline Beeghly-Fadiel et al & 2008 & China & Asian & Breast & $1079 / 1082$ & 0.236 & (7) \\
\hline Tao et al & 2010 & China & Asian & Cervical & $225 / 170$ & 0.169 & $(31)$ \\
\hline Yаo & 2011 & China & Asian & Cervical & $95 / 120$ & 0.326 & (33) \\
\hline Zhang et al & 2010 & China & Asian & Epithelial ovarian & $130 / 159$ & 0.713 & $(32)$ \\
\hline Malik et al & 2011 & India & Asian & Gastric & $108 / 195$ & 0.547 & (14) \\
\hline Malik et al & 2011 & India & Asian & Esophageal & $135 / 195$ & 0.547 & $(15)$ \\
\hline \multirow[t]{3}{*}{ Zhang et al } & 2005 & China & Asian & Gastric & $201 / 350$ & 0.888 & $(26)$ \\
\hline & & & & NSCLC & $243 / 350$ & 0.888 & \\
\hline & & & & ESCC & $258 / 350$ & 0.888 & \\
\hline
\end{tabular}

HWE, Hardy-Weinberg equilibrium; NSCLC, non-small-cell lung cancer; ESCC, esophageal squamous cell carcinoma.

studies, it was classified in the 'other' group) and smoking status. Two methods were utilized: the dominant genetic model (GG/GA vs. AA) and allelic contrast (G allele vs. A allele).

The between-study heterogeneity was assessed through Chi-square-based Q test (Cochran's Q statistic) and $\mathrm{I}^{2}$ statistic $(9,10)$. A fixed-effects model (the Mantel-Haenszel method) was used when $\mathrm{P}>0.05$ and $\mathrm{I}^{2}<50 \%$ (11). Otherwise, the random-effects model [the DerSimonian and Laird (12) method] was used. Additionally, meta-regression and sensitivity analyses were performed to investigate the sources of heterogeneity and assess the stability of the results, respectively. The Hardy-Weinberg equilibrium (HWE) in controls was recalculated and $\mathrm{P}<0.05$ was considered to indicate a statistically significant difference. The Begg's rank correlation method (13) was used to investigate publication bias. All analyses were performed with Stata software version 12.0 (StataCorp LP, College Station, TX, USA).

\section{Results}

Study characteristics. After comprehensive searching, 25 eligible publications $(6,7,14-36)$ including 27 studies with a total of 6,166 cases and 7,195 controls, were included in this meta-analysis. Among these, Malik et al published 2 studies $(14,15)$ involving 2 different cancer types, using the same control populations. Additionally, Zhang et al (26) investigated 3 types of cancer in one publication. The genotype distribution of the controls in all the studies was consistent with Hardy-Weinberg equilibrium, except for 3 studies (34-36). Twenty studies used the polymerase chain reaction-restriction fragment length polymorphism method to analyse genotypes. The cases were histopathologically diagnosed in the majority of the studies and the controls were free from cancer. The characteristics of studies on MMP7-181A/G polymorphism are provided in Table I and the flow diagram of the literature search is shown in Fig. 1.

Quantitative synthesis. The MMP7-181G allele frequency varied widely among the control subjects of the 27 case-control studies, ranging from 0.028 in Asian to 0.57 in European populations.

For the overall analysis of the 27 case-control studies, a significantly increased cancer risk was found to be associated with the $\mathrm{G}$ allele compared to the A allele ( $\mathrm{OR}=1.34$, 
Table II. Stratified analyses of the effect of metalloproteinase 7-181A/G polymorphism on cancer risk (HWE/HWD groups).

\begin{tabular}{|c|c|c|c|c|c|c|}
\hline \multirow[b]{2}{*}{ Variables } & \multirow[b]{2}{*}{ No. ${ }^{a}$} & \multirow[b]{2}{*}{ Cases/controls } & \multicolumn{2}{|c|}{ GG/GA vs. AA } & \multicolumn{2}{|c|}{ G vs. A } \\
\hline & & & OR $(95 \% \mathrm{CI})$ & P-value ${ }^{b}$ & OR $(95 \% \mathrm{CI})$ & P-value ${ }^{b}$ \\
\hline Total & 27 & $6166 / 7195$ & $1.33(1.15-1.54)$ & $<0.001$ & $1.34(1.17-1.53)$ & $<0.001$ \\
\hline \multicolumn{7}{|l|}{ Cancer type } \\
\hline Gastric & 5 & $886 / 1250$ & $1.34(0.82-2.20)$ & 0.002 & $1.41(0.97-2.03)$ & 0.007 \\
\hline Colorectal & 7 & $1487 / 1617$ & $1.02(0.76-1.36)$ & 0.042 & $1.05(0.85-1.32)$ & 0.036 \\
\hline Cervical & 4 & $687 / 642$ & $1.28(1.02-1.59)$ & 0.666 & 1.38 (1.16-1.63) & 0.799 \\
\hline Other & 11 & $3106 / 3686$ & $1.56(1.25-1.96)$ & 0.006 & $1.56(1.21-2.01)$ & $<0.001$ \\
\hline \multicolumn{7}{|c|}{ Quality assessment } \\
\hline Low & 12 & $2071 / 2409$ & $1.14(0.85-1.54)$ & $<0.001$ & $1.18(0.89-1.56)$ & $<0.001$ \\
\hline High & 15 & $4095 / 4786$ & $1.42(1.23-1.65)$ & 0.059 & $1.34(1.17-1.53)$ & 0.011 \\
\hline \multicolumn{7}{|l|}{ Ethnicity } \\
\hline European & 5 & $1076 / 1170$ & $1.03(0.71-1.51)$ & 0.013 & $1.00(0.74-1.34)$ & 0.001 \\
\hline Asian & 21 & $4982 / 5912$ & $1.42(1.21-1.67)$ & 0.001 & $1.47(1.28-1.69)$ & 0.001 \\
\hline \multicolumn{7}{|c|}{ Source of control } \\
\hline HB & 16 & $3190 / 3975$ & $1.27(1.03-1.57)$ & $<0.001$ & $1.35(1.13-1.61)$ & $<0.001$ \\
\hline PB & 10 & $2976 / 3220$ & $1.40(1.14-1.73)$ & 0.028 & $1.32(1.05-1.65)$ & $<0.001$ \\
\hline \multicolumn{7}{|l|}{ Smoking status } \\
\hline Smoking & 7 & $651 / 498$ & $1.66(1.21-2.28)$ & 0.204 & $1.56(1.23-1.99)$ & 0.243 \\
\hline Non-smoking & 6 & $742 / 929$ & $1.57(1.15-2.14)$ & 0.399 & $1.59(1.25-2.00)$ & 0.895 \\
\hline
\end{tabular}

HWE/HWD, Hardy-Weinberg equilibrium/Hardy-Weinberg disequilibrium; OR, odds ratio; CI, confidence interval; HB, hospital-based; $\mathrm{PB}$, population-based; ${ }^{\mathrm{a}}$ number of comparisons; ${ }^{\mathrm{b}} \mathrm{P}$-value of Q-test for heterogeneity.

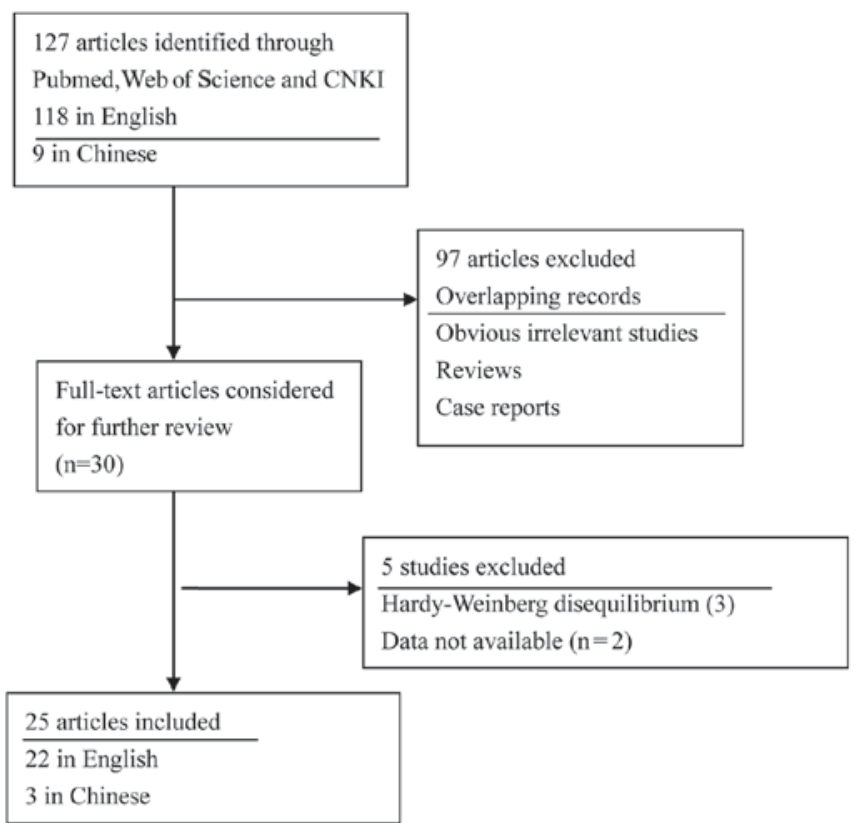

Figure 1. Flow diagram of the literature search in this meta-analysis. CNKI, China National Knowledge Infrastructure.

95\% CI: 1.17-1.53). A significantly increased cancer risk was also found to be associated with the $\mathrm{G}$ allele carriers (GG/GA genotypes) compared to the AA genotype in a dominant model (OR=1.33, 95\% CI: 1.15-1.54). Similarly, an increased cancer risk was identified in the 24 case-control studies under HWE [G vs. A: OR=1.43, 95\% CI: 1.26-1.62 (Table III); GG/GA vs. AA: OR=1.42, 95\% CI: $1.23-1.63)$ ].

Data were stratified according to the ethnicity of the participants into European, Asian and other. A statistically significant association was observed with the Asian but not the European ethnicity, regardless of whether studies under Hardy-Weinberg disequilibrium (HWD) were excluded (Tables II and III).

Data were stratified according to cancer type into gastric, cervical, colorectal and other types of cancer (Tables II and III). A significant association was observed between MMP7-181A/G polymorphism and cervical and other types of cancer, regardless of whether studies under HWD were excluded. Of note, certain inconsistencies were oserved in the HWE and HWE/HWD groups. In the former, a significantly elevated risk for gastric cancer was observed $(\mathrm{OR}=1.72,95 \% \mathrm{CI}: 1.33-2.23$ for $\mathrm{GG} / \mathrm{GA}$ vs. $\mathrm{AA}$; $\mathrm{OR}=1.67$, 95\% CI: $1.35-2.08$ for G vs. A), whereas no significant association with gastric cancer was observed in the latter $(\mathrm{OR}=1.34,95 \% \mathrm{CI}: 0.82-2.20$ for $\mathrm{GG} / \mathrm{GA}$ vs. $\mathrm{AA} ; \mathrm{OR}=1.41$, 95\% CI: $0.97-2.03$ for $\mathrm{G}$ vs. A). Additionally, no increased colorectal cancer risk was found to be associated with the $\mathrm{G}$ allele or $\mathrm{G}$ allele carriers in the HWE or the HWE/HWD group.

When smoking status was considered, a significantly increased cancer risk was found to be associated with the $\mathrm{G}$ allele and $\mathrm{G}$ allele carriers in the smoking as well as the 
Table III. Stratified analyses of the effect of metalloproteinase 7-181A/G polymorphism on cancer risk (Hardy-Weinberg equilibrium group).

\begin{tabular}{|c|c|c|c|c|c|c|}
\hline \multirow[b]{2}{*}{ Variables } & \multirow[b]{2}{*}{ No. ${ }^{a}$} & \multirow[b]{2}{*}{ Cases/controls } & \multicolumn{2}{|c|}{ GG/GA vs. AA } & \multicolumn{2}{|c|}{ G vs. A } \\
\hline & & & OR $(95 \% \mathrm{CI})$ & $\mathrm{P}^{\mathrm{b}}$ & OR $(95 \% \mathrm{CI})$ & $\mathrm{P}^{\mathrm{b}}$ \\
\hline Total & 24 & $5711 / 6716$ & $1.42(1.23-1.63)$ & 0.001 & $1.43(1.26-1.62)$ & $<0.001$ \\
\hline \multicolumn{7}{|l|}{ Cancer type } \\
\hline Gastric & 4 & $807 / 1081$ & $1.72(1.33-2.23)$ & 0.691 & $1.67(1.35-2.08)$ & 0.925 \\
\hline Colorectal & 7 & $1487 / 1617$ & $1.02(0.76-1.36)$ & 0.042 & $1.05(0.85-1.32)$ & 0.036 \\
\hline Cervical & 3 & $470 / 452$ & $1.40(1.07-1.84)$ & 0.961 & 1.45 (1.18-1.77) & 0.913 \\
\hline Other & 10 & $2947 / 3566$ & $1.64(1.30-2.07)$ & 0.007 & $1.68(1.36-2.08)$ & 0.003 \\
\hline \multicolumn{7}{|c|}{ Quality assessment } \\
\hline Low & 9 & $1616 / 1930$ & $1.31(0.94-1.84)$ & 0.001 & $1.37(1.01-1.86)$ & 0.001 \\
\hline High & 15 & $4095 / 4786$ & $1.42(1.23-1.65)$ & 0.059 & $1.41(1.24-1.61)$ & 0.011 \\
\hline \multicolumn{7}{|l|}{ Ethnicity } \\
\hline European & 3 & $838 / 881$ & $1.30(0.91-1.86)$ & 0.135 & $1.24(0.97-1.59)$ & 0.091 \\
\hline Asian & 20 & $4765 / 5722$ & $1.45(1.23-1.71)$ & 0.001 & $1.49(1.29-1.72)$ & 0.001 \\
\hline \multicolumn{7}{|l|}{ Source of control } \\
\hline $\mathrm{HB}$ & 15 & $3111 / 3806$ & 1.35 (1.11-1.64) & 0.003 & $1.41(1.19-1.67)$ & $<0.001$ \\
\hline PB & 8 & $2542 / 2799$ & $1.54(1.21-1.98)$ & 0.031 & $1.45(1.18-1.79)$ & 0.029 \\
\hline \multicolumn{7}{|l|}{ Smoking status } \\
\hline Smoking & 7 & $651 / 498$ & $1.66(1.21-2.28)$ & 0.204 & $1.56(1.23-1.99)$ & 0.243 \\
\hline Non-smoking & 6 & $742 / 929$ & $1.57(1.15-2.14)$ & 0.399 & $1.59(1.25-2.00)$ & 0.895 \\
\hline
\end{tabular}

OR, odds ratio; CI, confidence interval; HB, hospital-based; PB, population-based; anumber of comparisons; ${ }^{\text {b}} \mathrm{P}$-value of Q-test for heterogeneity.

non-smoking groups. The stratified analyses of the eligible studies are summarized in Tables II and III.

Evaluation of heterogeneity. In the 27 case-control studies, it was observed that variable ethnicity could explain $34.82 \%$ (G vs. A) and $13.54 \%$ (GG/GA vs. AA) of the $\mathrm{I}^{2}$, whereas HWE could explain $43.93 \%$ (G vs. A) and $35.28 \%$ (GG/GA vs. AA) of the $\mathrm{I}^{2}$. In the 24 case-control studies under HWE, none of these variables contributed significantly to heterogeneity.

Sensitivity analysis. A sensitivity analysis was conducted to evaluate the effect of each individual study on the pooled OR and removal of any individual study imparted no significant difference in the HWE or the HWE/HWD group.

Publication bias analysis. No evidence of publication bias for the association of MMP7-181A/G polymorphism with cancer risk was identified in our meta-analysis (HWE group, $\mathrm{P}=0.90$ for $\mathrm{GG} / \mathrm{GA}$ vs. $\mathrm{AA}$ and $\mathrm{P}=0.07$ for $\mathrm{G}$ vs. $\mathrm{A}$; HWE/HWD group, $\mathrm{P}=0.97$ for $\mathrm{GG} / \mathrm{GA}$ vs. $\mathrm{AA}$ and $\mathrm{P}=0.09$ for $\mathrm{G}$ vs. $\mathrm{A}$ ).

\section{Discussion}

Our meta-analysis was a systematic review of the association between the MMP7-181A/G polymorphism and cancer susceptibility, which provided evidence that the $\mathrm{G}$ allele and $\mathrm{G}$ allele carriers were significantly associated with an increased cancer risk (also in Asian ethnicity when race was considered). Data were then stratified by cancer type. A significantly increased risk of cervical and other types of cancer were observed in the HWE and HWE/HWD groups (GG/GA vs. AA; G vs. A) and an increased risk of gastric cancer was observed in the HWE group (GG/GA vs. AA; G vs. A). However, we failed to identify any significant association between MMP7-181A/G polymorphism and gastric cancer in the HWE/HWD group. Therefore, we hypothesized that the results of the 24 case-control studies under HWE were reliable. However, further studies on the effect of MMP7-181A/G polymorphism on cancer risk are required to support this hypothesis.

Data were also stratified according to European and Asian ethnicity. A statistically significant association was observed in the Asian but not in the European populations. Although the reason for this discrepancy is unclear, it may be attributed to differences in genetic traits among different ethnic groups and a potential reporting bias.

Tobacco is a well-known carcinogen, exposure to which may lead to smoking-related cancers. Increased cancer susceptibility was found to be associated with the $G$ allele and $\mathrm{G}$ allele carriers in the smoking as well as the non-smoking groups. These findings indicate that MMP7-181A/G polymorphism may play a pivotal role in cancer development.

Several limitations should be considered in our meta-analysis. First, the majority of the eligible studies only addressed the association of MMP7-181A/G polymorphism with cancer risk. 
However, we believe that further studies assessing the effect of gene-gene or gene-environment interactions may eventually achieve a more comprehensive understanding. Second, publication bias is likely to exist, despite the publication bias of low significance or lack thereof in our meta-analysis. Third, significant heterogeneity was detected in the overall comparisons and some of the subgroup analyses. Therefore, a meta-regression analysis was performed to identify the sources of heterogeneity and it was observed that HWE and ethnicity could explain the heterogeneity across studies, whereas other variables, such as cancer type and source of controls could not, possibly due to the heterogeneity resulting from other factors.

In conclusion, the MMP7-181A/G polymorphism is a risk factor for cancer development, particularly cervical and other types of cancer, in Asian populations. Further case-control studies (including HWE or HWE/HWD) estimating the effect of gene-gene and gene-environment interactions may eventually achieve a more comprehensive understanding.

\section{References}

1. Wolf K and Friedl P: Mapping proteolytic cancer cell-extracellular matrix interfaces. Clin Exp Metastasis 26: 289-298, 2009.

2. Chakraborti S, Mandal M, Das S, Mandal A and Chakraborti T: Regulation of matrix metalloproteinases: an overview. Mol Cell Biochem 253: 269-285, 2003.

3. Malemud CJ: Matrix metalloproteinases (MMPs) in health and disease: an overview. Front Biosci 11: 1696-1701, 2006

4. VanSaun MN and Matrisian LM: Matrix metalloproteinases and cellular motility in development and disease. Birth Defects Res C Embryo Today 78: 69-79, 2006.

5. Wilson CL and Matrisian LM: Matrilysin: an epithelial matrix metalloproteinase with potentially novel functions. Int J Biochem Cell Biol 28: 123-136, 1996.

6. Lu Z, Wang Y,Zhang Q, et al: Association between the functional polymorphism in the matrix metalloproteinase-7 promoter and susceptibility to adult astrocytoma. Brain Res 1118: 6-12, 2006.

7. Beeghly-Fadiel A, Long JR, Gao YT, et al: Common MMP-7 polymorphisms and breast cancer susceptibility: a multistage study of association and functionality. Cancer Res 68: 6453-6459, 2008.

8. Woolf B: On estimating the relation between blood group and disease. Ann Hum Genet 19: 251-253, 1955.

9. Higgins JP, Thompson SG, Deeks JJ and Altman DG: Measuring inconsistency in meta-analyses. BMJ 327: 557-560, 2003.

10. Cochran WG: The combination of estimates from different experiments. Biometrics 10: 101-129, 1954.

11. Mantel N and Haenszel W: Statistical aspects of the analysis of data from retrospective studies of disease. J Natl Cancer Inst 22: 719-748, 1959.

12. DerSimonian R and Kacker R: Random-effects model for meta-analysis of clinical trials: an update. Contemp Clin Trials 28: 105-114, 2007.

13. Begg CB and Mazumdar M: Operating characteristics of a rank correlation test for publication bias. Biometrics 50: 1088-1101, 1994.

14. Malik MA, Zargar SA and Mittal B: Role of the metalloproteinase-7 $(181 \mathrm{~A}>\mathrm{G})$ polymorphism in gastric cancer susceptibility: a case control study in Kashmir valley. Asian Pac J Cancer Prev 12: 73-76, 2011.

15. Malik MA, Sharma KL, Zargar SA and Mittal B: Association of matrix metalloproteinase-7 $(-181 \mathrm{~A}>\mathrm{G})$ polymorphism with risk of esophageal squamous cell carcinoma in Kashmir Valley. Saudi J Gastroenterol 17: 301-306, 2011

16. Sugimoto M, Furuta T, Kodaira C, et al: Polymorphisms of matrix metalloproteinase-7 and chymase are associated with susceptibility to and progression of gastric cancer in Japan. J Gastroenterol 43: 751-761, 2008.
17. Dziki L, Przybylowska K, Majsterek I, Trzcinski R, Mik M and Sygut A: A/G polymorphism of the MMP-7 gene promoter region in colorectal cancer. Pol Przegl Chir 83: 622-626, 2011.

18. Fang WL, Liang WB, He H, et al: Association of matrix metalloproteinases 1,7 , and 9 gene polymorphisms with genetic susceptibility to colorectal carcinoma in a Han Chinese population. DNA Cell Biol 29: 657-661, 2010.

19. Srivastava P, Gangwar R, Kapoor R and Mittal RD: Bladder cancer risk associated with genotypic polymorphism of the matrix metalloproteinase-1 and 7 in North Indian population. Dis Markers 29: 37-46, 2010.

20. Lievre A, Milet J, Carayol J, et al: Genetic polymorphisms of MMP1, MMP3 and MMP7 gene promoter and risk of colorectal adenoma. BMC Cancer 6: 270, 2006.

21. Yi YC, Chou PT, Chen LY, et al: Matrix metalloproteinase-7 (MMP-7) polymorphism is a risk factor for endometrial cancer susceptibility. Clin Chem Lab Med 48: 337-344, 2010.

22. Singh H, Jain M and Mittal B: MMP-7 (-181A>G) promoter polymorphisms and risk for cervical cancer. Gynecol Oncol 110: 71-75, 2008.

23. Qiu W, Zhou G, Zhai Y, et al: No association of MMP-7, MMP-8, and MMP-21 polymorphisms with the risk of hepatocellular carcinoma in a Chinese population. Cancer Epidemiol Biomarkers Prev 17: 2514-2518, 2008.

24. Ohtani H, Maeda N and Murawaki Y: Functional polymorphisms in the promoter regions of matrix metalloproteinase-2, -3, -7, -9 and TNF-alpha genes, and the risk of colorectal neoplasm in Japanese. Yonago Acta Medica 52: 47-56, 2009.

25. Woo M, Park K, Nam J and Kim JC: Clinical implications of matrix metalloproteinase- $1,-3,-7,-9,-12$, and plasminogen activator inhibitor-1 gene polymorphisms in colorectal cancer. J Gastroenterol Hepatol 22: 1064-1070, 2007.

26. Zhang J, Jin X, Fang S, et al: The functional polymorphism in the matrix metalloproteinase-7 promoter increases susceptibility to esophageal squamous cell carcinoma, gastric cardiac adenocarcinoma and non-small cell lung carcinoma. Carcinogenesis 26: 1748-1753, 2005.

27. Ghilardi G, Biondi ML, Erario M, Guagnellini E and Scorza R: Colorectal carcinoma susceptibility and metastases are associated with matrix metalloproteinase-7 promoter polymorphisms. Clin Chem 49: 1940-1942, 2003.

28. de Lima JM, de Souza LG, da Silva ID and Forones NM: E-cadherin and metalloproteinase- 1 and -7 polymorphisms in colorectal cancer. Int J Biol Markers 24: 99-106, 2009.

29. Li Y, Jin X, Kang S, et al: Polymorphisms in the promoter regions of the matrix metalloproteinases-1, $-3,-7$, and -9 and the risk of epithelial ovarian cancer in China. Gynecol Oncol 101: 92-96, 2006.

30. Li JY, Tian M and Zhao A: Polymorphism in the promoter region of the metalloproteinase-7 increases susceptibility and risk of metastasis of gastric adenocarcinoma. Gastroenterology 134: A603, 2008.

31. Tao HJ, Wu SH, Zhang L, et al: Correlation of polymorphism of IL-8 and MMP-7 genes with the risk of early stage cervical cancer in Shanxi. Chin Remedies and Clinics 10: 852-854, 2010.

32. Zhang Y, Du H, Kang S, Sun DL and Li Y: Association of SNP in matrix metalloproteinase-7 polymorphism with susceptibility of ovarian cancer. China J Mod Med 20: 2792-2798, 2010.

33. Yao N: Correlation of polymorphism of MMP-7 gene with the risk of early stage cervical cancer. China J Modern Med 21: 3519-3521, 2011 (In Chinese).

34. Kubben FJ, Sier CF, Meijer MJ, et al: Clinical impact of MMP and TIMP gene polymorphisms in gastric cancer. Br J Cancer 95: 744-751, 2006.

35. Vairaktaris E, Serefoglou Z, Yapijakis C, et al: High gene expression of matrix metalloproteinase-7 is associated with early stages of oral cancer. Anticancer Res 27: 2493-2498, 2007.

36. Wu S, Lu S, Tao H, et al: Correlation of polymorphism of IL-8 and MMP-7 with occurrence and lymph node metastasis of early stage cervical cancer. J Huazhong Univ Sci Technolog Med Sci 31: 114-119, 2011. 